\title{
Direct comparison of two extended half-life PEGylated recombinant FVIII products: a randomized, crossover pharmacokinetic study in patients with severe hemophilia A
}

\author{
Alexander Solms ${ }^{1}(\mathbb{D}) \cdot$ Anita Shah $^{2} \cdot$ Erik Berntorp $^{3} \cdot$ Andreas Tiede $^{4} \cdot$ Alfonso lorio $^{5,6} \cdot$ Camila Linardi $^{2}$. \\ Maurice Ahsman ${ }^{7}$ - Maria Elisa Mancuso $^{8} \cdot$ Tihomir Zhivkov $^{9} \cdot$ Toshko Lissitchkov $^{9}$
}

Received: 10 July 2020 / Accepted: 17 September 2020 / Published online: 24 September 2020

(C) The Author(s) 2020

\begin{abstract}
An open-label, crossover randomized study was performed to compare the pharmacokinetics (PK) of damoctocog alfa pegol and rurioctocog alfa pegol, two recombinant factor VIII (FVIII) products indicated in patients with hemophilia A, both conjugated to polyethylene glycol to reduce clearance and extend time in circulation. Adult patients $(N=18)$ with severe hemophilia $\mathrm{A}(\mathrm{FVIII}<$ $1 \mathrm{IU} / \mathrm{dL}$ ), previously treated with any FVIII product for $\geq 150$ exposure days, were randomized to receive a single $50 \mathrm{IU} / \mathrm{kg}$ infusion of damoctocog alfa pegol followed by rurioctocog alfa pegol, or vice versa, with $\geq 7$-day washout between doses. FVIII activity was measured using the one-stage clotting assay. PK parameters, including area under the curve from time 0 to the last data point $\left(\mathrm{AUC}_{0-\text { tlast }}\right.$, primary parameter), dose-normalized $\mathrm{AUC}\left(\mathrm{AUC}_{\mathrm{norm}}\right)$, and time to threshold, were calculated based on 11 time points between 0.25 and $120 \mathrm{~h}$ post-dose and evaluated using a noncompartmental model. Due to differences in batchspecific vial content used for the study, actual administered median doses were $54.3 \mathrm{IU} / \mathrm{kg}$ for damoctocog alfa pegol and 61.4 $\mathrm{IU} / \mathrm{kg}$ for rurioctocog alfa pegol. Based on actual dosing, a significantly higher geometric mean (coefficient of variation [\% CV]) $\mathrm{AUC}_{\text {norm }}$ was observed for damoctocog alfa pegol $(43.8 \mathrm{~h} \mathrm{~kg} / \mathrm{dL}$ [44.0]) versus rurioctocog alfa pegol $(36.0 \mathrm{~h} \mathrm{~kg} / \mathrm{dL}[40.1, P<$ $0.001]$ ). Based on population PK modeling, median time to reach $1 \mathrm{IU} / \mathrm{dL}$ was $16 \mathrm{~h}$ longer for damoctocog alfa pegol compared with rurioctocog alfa pegol. No adverse events or any immunogenicity signals were observed. Overall, damoctocog alfa pegol had a superior PK profile versus rurioctocog alfa pegol. Trial registration number: NCT04015492 (ClinicalTrials.gov identifier). Date of registration: July 9, 2019
\end{abstract}

Keywords Factor VIII · Extended half-life $\cdot$ Hemophilia A $\cdot$ PEGylated $\cdot$ Head-to-head study $\cdot$ Population pharmacokinetics

Electronic supplementary material The online version of this article (https://doi.org/10.1007/s00277-020-04280-3) contains supplementary material, which is available to authorized users.

Alexander Solms

alexander.solms@bayer.com

1 Clinical Pharmacometrics, Pharmaceuticals Research \& Development, Bayer AG, 13353 Berlin, Germany

2 Bayer, Whippany, NJ, USA

3 Centre for Thrombosis and Haemostasis, Lund University, Skåne University Hospital, Malmö, Sweden

4 Department of Hematology, Hemostasis, Oncology and Stem Cell Transplantation, Hannover Medical School, Hanover, Germany
5 McMaster-Bayer Endowed Research Chair in Clinical Epidemiology of Congenital Bleeding Disorders, Department of Medicine, McMaster University, Hamilton, Canada

6 Department of Health Research Methods, Evidence and Impact, McMaster University, Hamilton, Canada

7 LAP\&P Consultants BV, Leiden, the Netherlands

8 Center for Thrombosis and Hemorrhagic Diseases, Humanitas Clinical and Research Center - IRCCS, Rozzano, Milan, Italy

9 Specialized Hospital for Active Treatment, Sofia, Bulgaria 


\section{Introduction}

In patients with severe hemophilia A (factor VIII [FVIII] levels $<1 \mathrm{IU} / \mathrm{dL}$ ), prophylaxis with FVIII replacement therapy remains the standard of care [1] and is associated with a reduction in bleeding, including joint bleeds, thereby protecting patients from further complications associated with recurrent bleeds such as joint disease and disability $[1,2]$. However, standard replacement recombinant FVIII (rFVIII) products have a short half-life (8-12 h) and, consequently, require frequent infusions to maintain FVIII plasma levels and provide optimal bleeding control $[1,3]$. Such frequent dosing is associated with a significant treatment burden and may cause venous access issues or lead to inefficient prophylaxis due to poor patient adherence [4-7].

Extended half-life (EHL) rFVIII products with improved pharmacokinetic (PK) profiles compared with standard halflife (SHL) rFVIII products have the potential to offer longer treatment intervals or to achieve higher trough levels $[8,9]$. Covalent attachment of polyethylene glycol (PEG; PEGylation) and fusion with the fragmented crystallizable (Fc) portion of the immunoglobulin (Ig) G1 molecule have been utilized to modify rFVIII to extend its half-life $[9,10]$.

Damoctocog alfa pegol (BAY 94-9027, Jivi®, Bayer, Germany) and rurioctocog alfa pegol (BAX 855; Adynovate $\AA_{\text {Adynovi }}{ }^{\circ}$; Takeda, Japan) are two EHL rFVIII products that both utilize the attachment of PEG as modification to extend their half-life although the size of the PEG moiety used, site of PEGylation, and FVIII truncation differ between them $[11,12]$. Damoctocog alfa pegol is a Bdomain-deleted (BDD) rFVIII product that has been sitespecifically PEGylated at a single amino acid site with a branched $60 \mathrm{kDa}$ PEG molecule to extend its half-life [11]. Rurioctocog alfa pegol is a full-length $\mathrm{rFVIII}$ product that has been PEGylated at its B-domain, with a branched 20-kDa PEG molecule (Advate ${ }^{\circledR}$; Baxter, USA) [12, 13]. Rurioctocog alfa pegol was first licensed in 2015, and damoctocog alfa pegol in 2018, for the treatment of hemophilia $\mathrm{A}$, based on efficacy and safety data from their respective pivotal phase 2/3 clinical trials [14-19].

Both damoctocog alfa pegol and rurioctocog alfa pegol have demonstrated improvements in their respective PK profiles compared with SHL rFVIII products $[17,20,21]$. In previously treated patients with severe hemophilia A, damoctocog alfa pegol demonstrated an $~ 1$.4-fold increase in half-life $\left(t_{1 / 2}\right)$ and dose-normalized area under the curve compared with a SHL rFVIII product (sucrose-formulated rFVIII [Kogenate ${ }^{\circledR}$ FS, Bayer, USA]) [20, 21]. For rurioctocog alfa pegol, an $\sim 1$.4-fold increase in $t_{1 / 2}$ and an $\sim$ 1.9-fold increase in area under the curve from time 0 to infinity $\left(\mathrm{AUC}_{0-\infty}\right)$ compared with a conventional $\mathrm{rFVIII}$ (Advate ${ }^{\circledR}$ ) were confirmed in the phase 2/3 PROLONGATE study [17]. Indirect comparisons of damoctocog alfa pegol and rurioctocog alfa pegol using PK data from such studies may be inaccurate and inconclusive, for example, because of interpatient heterogeneity in PK profiles, differences between the studies in the doses and assays used, and in analysis methodologies. Indeed, direct comparisons of PK between different concentrates should ideally be evaluated through a crossover study design to minimize the potential for confounding [22-24].

To date, only one head-to-head crossover PK study of EHL rFVIII products has been reported in hemophilia A. In this study by Shah et al., improvements in several PK parameters following a single infusion of damoctocog alfa pegol were demonstrated in patients with severe hemophilia A, compared with rFVIII Fc fusion protein $\left(\mathrm{rFVIIIFc}\right.$; Elocta ${ }^{\circledR}$; Swedish Orphan Biovitrum Ltd, UK) [25]. In a noncrossover, realworld study conducted in Canada, the PK profiles of rurioctocog alfa pegol and $\mathrm{rFVIIIFc}$ were shown to be almost identical in 25 adolescents aged 12-18 years switching from rFVIIIFc to rurioctocog alfa pegol prophylaxis [26]. However, to date, no studies have been performed to compare the PK of two PEGylated EHL rFVIII products. The objective of this study was to directly compare the PK profiles of the two PEGylated EHL rFVIII products, damoctocog alfa pegol and rurioctocog alfa pegol in patients with severe hemophilia A, using a head-to-head, randomized crossover study design. In addition, population PK modeling was performed to explore any differences between damoctocog alfa pegol and rurioctocog alfa pegol in the simulated time to FVIII threshold levels.

\section{Methods}

\section{Study design}

This was a single-center, randomized, open-label, single-dose, crossover study (ClinicalTrials.gov identifier: NCT04015492) (Fig. 1). The study was conducted at the National Specialised Hospital for Active Treatment of Haematologic Diseases, in Sofia, Bulgaria. Enrolment began in August 2019 and the last patient to be enrolled completed the study in January 2020 . After a washout period of $\geq 3$ days or $\geq 5$ days for SHL or EHL FVIII products, respectively, patients were randomized $1: 1$ to receive a single infusion of $50 \mathrm{IU} / \mathrm{kg}$ damoctocog alfa pegol or $50 \mathrm{IU} / \mathrm{kg}$ rurioctocog alfa pegol, followed by a crossover to a single infusion of the other product, with 7-28-day washout period between doses to account for washout between treatments and patient schedules. Both products were administered as intravenous infusions of up to $10 \mathrm{~min}$. One batch was used for each study drug. Study drug doses were based on the nominal value on the label of the vial. The study was approved by the institutional review board at the single site and was carried out in compliance with the protocol, the 


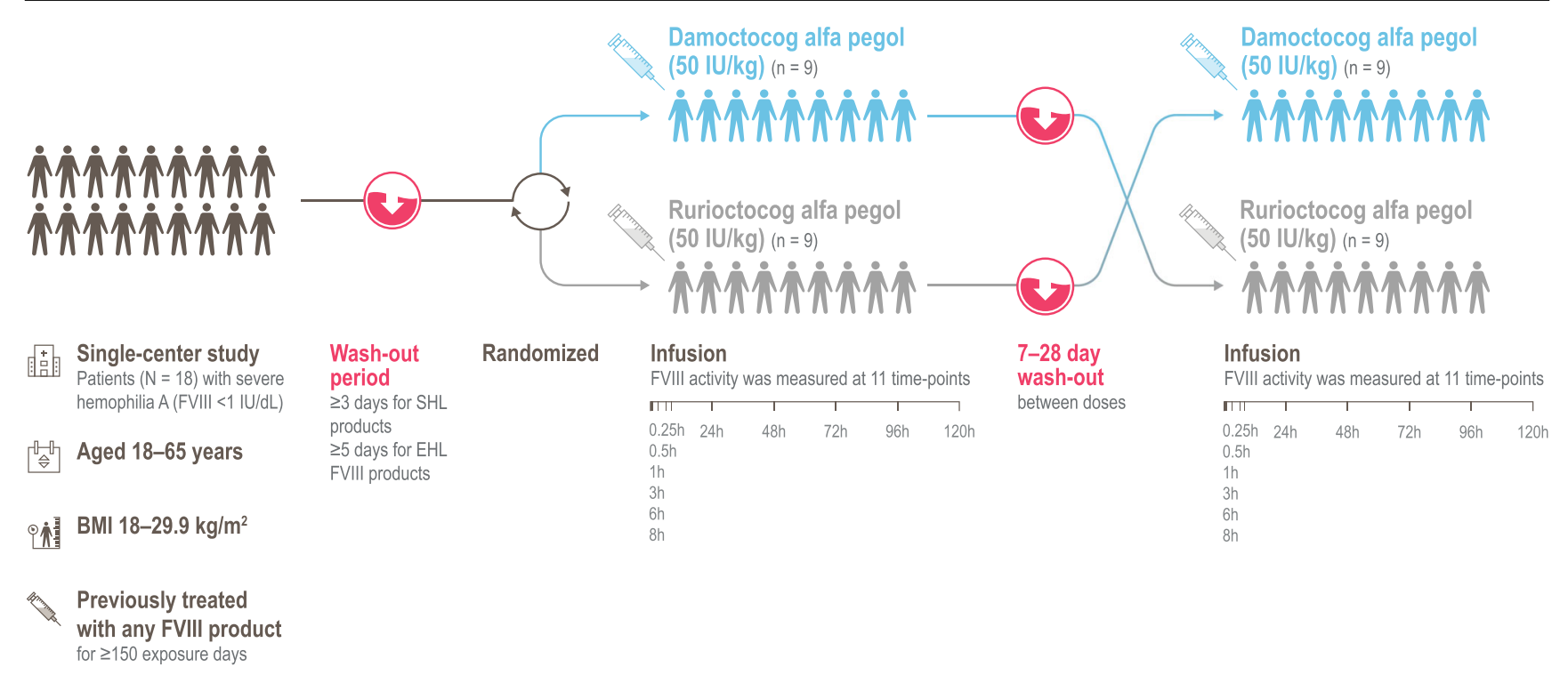

Fig. 1 Study design. BMI, body mass index; EHL, extended half-life; FVIII, factor VIII; SHL, standard half-life

principles of the 1975 Declaration of Helsinki and subsequent amendments, and good clinical practice guidelines. All patients gave written informed consent before initiation of any study-related procedures. A patient lay summary can be found in the Supplementary materials (Online resource 1).

\section{Patients}

Eligible patients were males aged 18-65 years with severe hemophilia A (FVIII $<1 \mathrm{IU} / \mathrm{dL})$ previously treated with any FVIII product for $\geq 150$ exposure days (EDs). Patients were required to have a body mass index of $18-29.9 \mathrm{~kg} / \mathrm{m}^{2}$ and were able to stop their current FVIII treatment to complete the wash-out period before study entry and between PK doses. Key exclusion criteria included the presence or history of a FVIII inhibitor ( $\geq 0.6$ Bethesda units $/ \mathrm{mL}$ ), diagnosis of any bleeding disorder other than hemophilia A, platelet count $<$ $75,000 / \mathrm{mm}^{3}$, HIV positive with a CD4 count of $<200 / \mathrm{mm}^{3}$, serum creatinine over twice the upper limit of normal (ULN), alanine aminotransferase or aspartate aminotransferase over 5 times ULN, or severe liver disease.

\section{Pharmacokinetic assessments}

Plasma samples were collected pre-dose and $0.25,0.5,1,3,6$, $8,24,48,72,96$, and $120 \mathrm{~h}$ after infusion of each drug. FVIII levels were measured using the same one-stage clotting assay that was validated for use for both damoctocog alfa pegol and rurioctocog alfa pegol (Online resource 2). Plasma concentrations of damoctocog alfa pegol and rurioctocog alfa pegol were determined by a turbidimetric assay with the SynthASil reagent and activated partial thromboplastin time (APTT) measured on the ACL Advance System against a calibration curve of standard human plasma. The following
PK parameters were assessed: AUC from time 0 to the last data point $\left(\mathrm{AUC}_{0-\text { tlast }}\right.$; primary $\mathrm{PK}$ parameter), $\mathrm{AUC}$; $\mathrm{AUC}$ normalized for actual dose per body weight $\left(\mathrm{AUC}_{\text {norm }}\right)$, maximum concentration $\left(C_{\max }\right)$, normalized $C_{\max }\left(C_{\max , \text { norm }}\right) ; t_{1 / 2}$; clearance (CL); mean residence time (MRT); volume of distribution at steady state $\left(V_{\mathrm{ss}}\right)$; and incremental recovery. The dose-normalized parameters were based on dosing adjusted for actual potency, as per the certificate of analysis for each product provided by the manufacturers. All parameters were calculated using noncompartmental analysis (NCA).

\section{Population PK model}

A single integrated population PK (popPK) model for damoctocog alfa pegol and rurioctocog alfa pegol was developed to simulate "time to reach" FVIII threshold levels of 1, 3, 5 , and $10 \mathrm{IU} / \mathrm{dL}$ for this study population. The analysis was based on data from all 18 participants.

A nonlinear mixed-effect modeling approach was utilized, as implemented in NONMEM ${ }^{\circledR}$ (version 7.4.1; ICON, Hanover, MD, USA). First, a structural model for each product was selected based on standard diagnostic tools, such as raw-data inspection, goodness of fit, and precision of parameter estimates. As suggested by previous analyses, potential candidates were one- or two-compartment models parameterized in terms of CL and central volume $(\mathrm{Vc})$ and, for the twocompartment model, peripheral volume (Vp) and intercompartmental clearance $(Q)$. Residual (unexplained) variability was described using a combined (proportional and additive) error model. Data below the lower limit of quantification (LLOQ) were accounted for using the M3 method [27]. Next, an integrated model was developed by combining the two structural models and subsequently refining the model by testing whether damoctocog alfa pegol and rurioctocog alfa 
pegol have statistically significant differences in PK parameters (e.g., CL) using the likelihood ratio test (LRT) and a $P$ value of 0.01 . Because of the small study size, no additional covariate search was conducted. The popPK model was qualified using standard model diagnostic tools, such as uncertainty in parameter estimates, plausibility of estimates (comparison with published information), goodness-of-fit plots, and visual predictive checks.

A sensitivity analysis was performed comparing the results of the approach described above with an alternative approach where previously reported popPK models for damoctocog alfa pegol [28] and rurioctocog alfa pegol [29] were employed (Online resource 3, Online resource Table 1).

\section{Safety}

Safety was assessed by means of clinical and laboratory evaluation at study visits and the recording of adverse events (AEs) and serious (S)AEs. All safety analyses were performed on the safety analysis set defined as those patients who received at least one dose of either damoctocog alfa pegol or rurioctocog alfa pegol. The study investigators reviewed all relevant AEs and SAEs and also assessed the intensity for each of these events. Laboratory analyses to test FVIII inhibitor and anti-PEG antibody development were also performed.

\section{Statistical analysis}

For statistical analysis of the PK parameters obtained by NCA, a log-normal distribution of the parameters was assumed. Log-transformed parameters were analyzed using analysis of variance (ANOVA), including sequence, patient, period, and treatment effects. Based on these analyses, point estimates (least square means), including confidence intervals (CIs, $90 \%$ and $95 \%$ ) for the damoctocog alfa pegol:rurioctocog alfa pegol ratio, were calculated The lower limit of the $90 \%$ CI for the ratio exceeding 0.8 would indicate that damoctocog alfa pegol is noninferior to rurioctocog alfa pegol in terms of PK; the lower limit of the 95\% CI for the ratio exceeding 1.0 would indicate that damoctocog alfa pegol is superior to rurioctocog alfa pegol. For PK parameters where a low value reflects an improved outcome, such as CL, the lower limit of the $95 \%$ CI for the ratio less than 1.0 would indicate that damoctocog alfa pegol is superior to rurioctocog alfa pegol. Safety analyses were descriptive.

\section{Results}

\section{Patients}

A total of 18 patients were randomized and received single doses of damoctocog alfa pegol and rurioctocog alfa pegol; the demographics and baseline characteristics of the patients are provided in Table 1. The median age of patients was 33.5 years.

\section{Dose adjustment and PK analyses}

The 50-IU/kg doses administered in this study were calculated based on the nominal potencies (1000 IU) as provided on the label of the vials which differed from that of the actual potencies, being $1030 \mathrm{IU} /$ vial for damoctocog alfa pegol and 1141 $\mathrm{IU} /$ vial for rurioctocog alfa pegol. This resulted in actual administered doses of approximately $3 \%$ and $14.1 \%$ higher than the planned 50-IU/kg doses for damoctocog alfa pegol and rurioctocog alfa pegol, respectively. In order to accurately compare the PK of the two products, the dose based on actual potency was considered in the analysis of the PK parameters. Based on the actual potencies, the administered median (range) dose was $54.3 \mathrm{IU} / \mathrm{kg}(51.5,56.5)$ for damoctocog alfa pegol and $61.4 \mathrm{IU} / \mathrm{kg}(57.1,65.3)$ for rurioctocog alfa pegol. For $\mathrm{AUC}_{0-\text { tlast }}$, the geometric mean value was $2311 \mathrm{~h} \mathrm{IU/dL}$ (percentage of coefficient of variation [\%CV], 44.0\%; 95\% CI, 1880-2850) following a dose of $54.3 \mathrm{IU} / \mathrm{kg}$ of damoctocog alfa pegol and $2150 \mathrm{~h} \mathrm{IU/dL}(\% \mathrm{CV}, 39.6 \%$; 95\% CI, 1780-2600) following a dose of $61.4 \mathrm{IU} / \mathrm{kg}$ of rurioctocog alfa pegol. Thus, a numerically higher geometric mean $\mathrm{AUC}_{0-\text { tlast }}$ was observed for damoctocog alfa pegol compared with rurioctocog alfa pegol for an approximately $10 \%$ lower actual dose of damoctocog alfa pegol compared with rurioctocog alfa pegol. For $\mathrm{AUC}_{0-\text { tlast }}$, the geometric least square mean for the damoctocog alfa pegol:rurioctocog

Table 1 Patient demographics and baseline characteristics

\begin{tabular}{ll}
\hline Characteristics & $\begin{array}{l}\text { Total } \\
N=18\end{array}$ \\
\hline Age, years & \\
Median (range) & $33.3(23,56)$ \\
Mean (SD) & $34.2(9.5)$ \\
Race, $n(\%)$ & \\
White & $18(100)$ \\
BMI, kg/m ${ }^{2}$ & \\
Median (range) & $24.45(18.1,29.8)$ \\
Mean (SD) & $23.76(4.25)$ \\
Previous FVIII replacement therapy, $n(\%)$ & $16(88.9)$ \\
On demand & $8(44.4)$ \\
Prophylaxis & $8(44.4)$ \\
Hemophilic arthropathy & $10(55.6)$ \\
Hepatitis viral infections, $n(\%)$ & $13(72.2)$ \\
Chronic hepatitis C & $13(72.2)$ \\
\hline
\end{tabular}

$B M I$, body mass index; FVIII, factor VIII; SD, standard deviation 
alfa pegol ratio was 1.0747 (90\% CI, 0.9958-1.1599; 95\% CI, 0.9796-1.1790).

Dose-normalized analyses considering the dosing based on actual potency were performed for an accurate and valid comparison of PK parameters between damoctocog alfa pegol and rurioctocog alfa pegol. The geometric mean $(\% \mathrm{CV})$ for $\mathrm{AUC}_{\text {norm }}$ was $43.8 \mathrm{~h} \mathrm{~kg} / \mathrm{dL}$ (44.0) for damoctocog alfa pegol and $36.0 \mathrm{~h} \mathrm{~kg} / \mathrm{dL}$ (40.1) for rurioctocog alfa pegol (Table 2). Out of 18 patients, $16(88.9 \%)$ showed a higher $\mathrm{AUC}_{\text {norm }}$ with damoctocog alfa pegol compared with rurioctocog alfa pegol (Fig. 2a). The geometric least square mean for the damoctocog alfa pegol:rurioctocog alfa pegol ratio was 1.22 , meeting the prespecified criteria for superiority $(95 \%$ CI $1.11-1.33, P=$ 0.0004 , Table 2). This superiority also corresponded to a significantly slower clearance of damoctocog alfa pegol, resulting in a prolonged $t_{1 / 2}$ versus rurioctocog alfa pegol. CL was significantly reduced for damoctocog alfa pegol compared with rurioctocog alfa pegol $(1.65 \mathrm{dL} / \mathrm{h},[1.33-2.06]$ versus $2.01 \mathrm{dL} / \mathrm{h},[1.64-2.46]), P<0.001$, Table 2 ), and a reduced clearance with damoctocog alfa pegol compared with rurioctocog alfa pegol was observed in $16(88.9 \%)$ out of 18 patients (Fig. 2b). The geometric mean [\% CV, 95\% CI] $t_{1 / 2}$ indicated a significantly longer $t_{1 / 2}$ for damoctocog alfa pegol $(17.0 \mathrm{~h},[37.9 \%, 14.1-20.4])$ versus rurioctocog alfa pegol (16.0 h, [39.0\%, 13.2-19.3], $P=0.0064$, Table 2). The prolonged $t_{1 / 2}$ was observed in $15(83.3 \%)$ out of 18 patients (Fig. 2c). Based on the individual data for all 18 patients, outcomes for $\mathrm{AUC}_{\mathrm{norm}}, \mathrm{CL}$, and $t_{1 / 2}$ were in favor of damoctocog alfa pegol in the majority of patients (Fig. 3). Additional PK parameters are provided in Table 2.

\section{Population PK modeling and time to threshold simulation}

A second distribution compartment could not be identified for damoctocog alfa pegol ( $\mathrm{CV}$ on $Q=103 \%)$; the PK of damoctocog alfa pegol was adequately described by a onecompartment model (technically, the PK of damoctocog alfa pegol was described by a two-compartment model fixing $Q$ to a very small value [0.001]), while a two-compartment model was used for rurioctocog alfa pegol. Compared with damoctocog alfa pegol, the $\mathrm{CL}$ of rurioctocog alfa pegol was significantly increased by $21 \%$ (95\% CI, 13-29). This effect was consistent across patients with an estimated betweenpatient variability of $11.5 \% \mathrm{CV}$ and 17 out of 18 patients having a CL value in favor of damoctocog alfa pegol compared with rurioctocog alfa pegol, respectively. The parameter estimates of the popPK model are shown in Table 3.

The PK model parameters and results for damoctocog alfa pegol are consistent with previous popPK analyses [25, 29].The median times to reach $1,3,5$, and $10 \mathrm{IU} / \mathrm{dL}$ were $16,11.8,9.4$, and $7.7 \mathrm{~h}$ longer for a dose of $50 \mathrm{IU} / \mathrm{kg}$, respectively, for damoctocog alfa pegol versus rurioctocog alfa pegol (Fig. 3). Sensitivity analyses using published population PK models provided similar results (Online resource 3). This suggests that the data from this study is consistent with PK

Table 2 Dose-normalized PK parameters following single-dose administrations of $54.3 \mathrm{IU} / \mathrm{kg}$ of damoctocog alfa pegol and $61.4 \mathrm{IU} / \mathrm{kg}$ of rurioctocog alfa pegol

\begin{tabular}{|c|c|c|c|c|c|}
\hline \multirow[t]{2}{*}{ PK parameter } & \multicolumn{2}{|c|}{ Geometric mean $(\% \mathrm{CV})(95 \% \mathrm{CI})$} & \multirow{2}{*}{$\begin{array}{l}\text { Ratio geometric least square mean ratio } \\
(95 \% \text { CI })\end{array}$} & \multirow{2}{*}{$\begin{array}{l}P \\
\text { value }\end{array}$} & \multirow{2}{*}{$\begin{array}{l}\text { In favor of damoctocog alfa } \\
\text { pegol } \\
N=18\end{array}$} \\
\hline & $\begin{array}{l}\text { Damoctocog alfa } \\
\text { pegol }\end{array}$ & $\begin{array}{l}\text { Rurioctocog alfa } \\
\text { pegol }\end{array}$ & & & \\
\hline $\begin{array}{l}\mathrm{AUC}_{\text {norm }}{ }^{\mathrm{b}}, \mathrm{kg} \\
\mathrm{h} / \mathrm{dL}\end{array}$ & $\begin{array}{l}43.8(44.0) \\
(35.5-54.0)\end{array}$ & $\begin{array}{l}36.0(40.1) \\
(29.7-43.7)\end{array}$ & $1.22(1.11-1.33)$ & 0.0004 & 16 \\
\hline$C_{\max }, \mathrm{b} g / \mathrm{dL}$ & $\begin{array}{l}1.99(24.3) \\
(1.76-2.24)\end{array}$ & $\begin{array}{l}1.85(28.6) \\
(1.61-2.12)\end{array}$ & $1.08(0.97-1.19)$ & 0.1464 & 14 \\
\hline $\mathrm{CL}^{\mathrm{b}}, \mathrm{dL} / \mathrm{h}$ & $\begin{array}{l}1.65(46.4) \\
(1.33-2.06)\end{array}$ & $\begin{array}{l}2.01(42.0) \\
(1.64-2.46)\end{array}$ & $0.82(0.75-0.90)$ & 0.0004 & 16 \\
\hline$t_{1 / 2}, \mathrm{~h}$ & $\begin{array}{l}17.0(37.9) \\
(14.1-20.4)\end{array}$ & $\begin{array}{l}16.0(39.0) \\
(13.2-19.3)\end{array}$ & $1.06(1.02-1.11)$ & 0.0064 & 15 \\
\hline MRTIV, h & $\begin{array}{l}24.59(37.6) \\
(20.5-29.5)\end{array}$ & $\begin{array}{l}22.81(37.1) \\
(19.1-27.3)\end{array}$ & $1.08(1.03-1.13)$ & 0.0039 & 13 \\
\hline$V_{\mathrm{ss}}^{\mathrm{b}}, \mathrm{dL}$ & $\begin{array}{l}40.7(16.2) \\
(37.6-44.1)\end{array}$ & $\begin{array}{l}45.8(15.8) \\
(42.4-49.6)\end{array}$ & $0.89(0.83-0.95)$ & 0.0024 & 12 \\
\hline $\begin{array}{l}\text { Incremental } \\
\text { recovery }\end{array}$ & $\begin{array}{l}1.91(25.2) \\
(1.69-2.16)\end{array}$ & $\begin{array}{l}1.71(33.1) \\
(1.46-2.01)\end{array}$ & $1.12(0.98-1.27)$ & 0.0856 & 14 \\
\hline
\end{tabular}

$A U C$, area under curve from 0 to infinity; $A U C_{n o r m}$, AUC normalized for actual dose per body weight; $C L$, clearance; $C_{\text {max }}$, maximum concentration, $C_{\max , \text { norm }}, C_{\max }$ normalized for actual dose per body weight; $t_{1 / 2}$, half-life; $M R T I V$, mean residence time after injection; $P K$, pharmacokinetic; $V_{s S}$, volume of distribution at steady state

${ }^{\text {a }}$ Ratio of damoctocog alfa pegol:rurioctocog alfa pegol

${ }^{\mathrm{b}}$ Based on actual doses (potency-adjusted) 
- - In favor of rurioctocog alfa pegol

$\square \longrightarrow$ In favor of damoctocog alfa pego
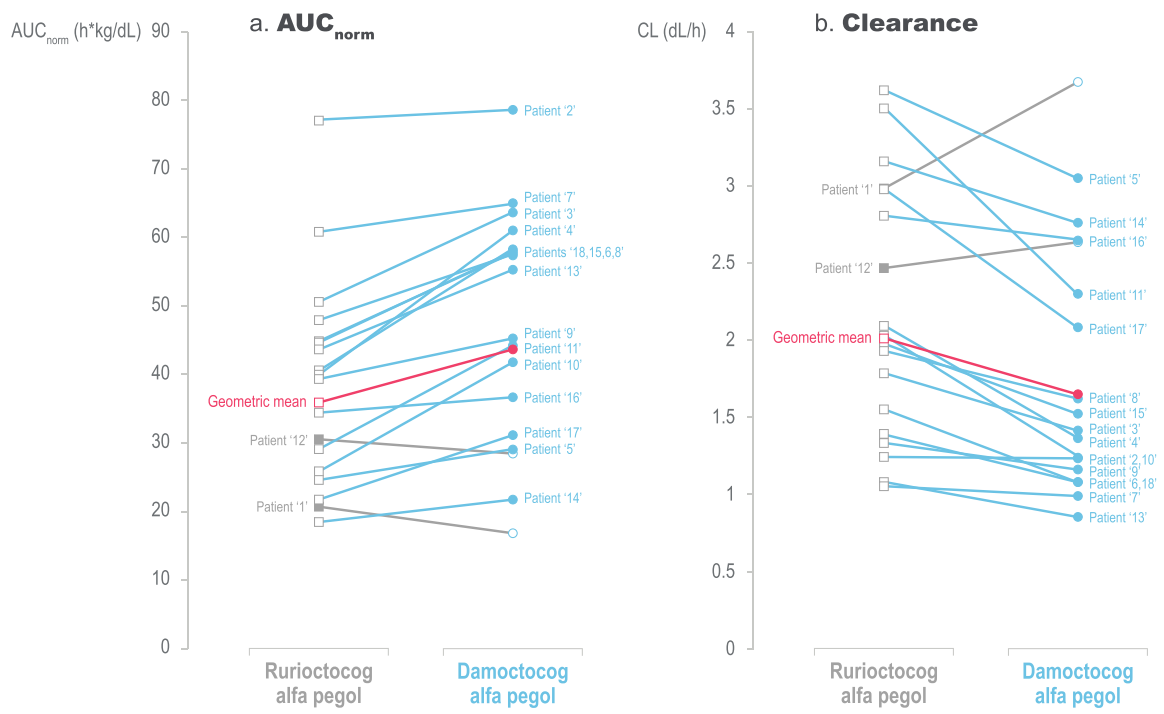

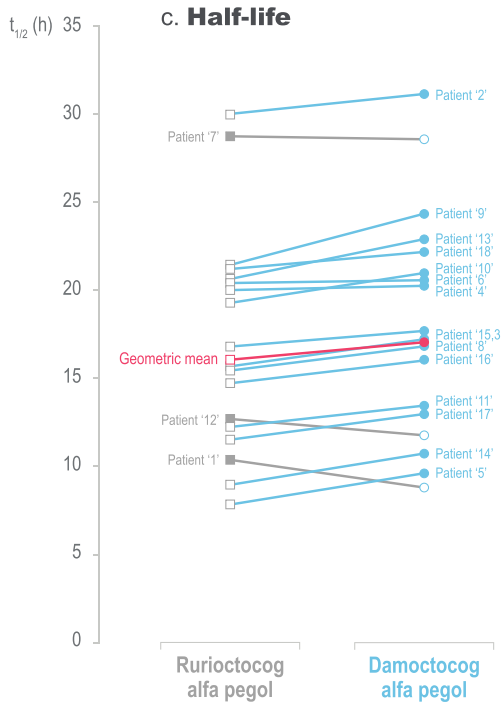

Fig. 2 Dose-normalized AUC, clearance, and half-life after a single infusion of damoctocog alfa pegol or rurioctocog alfa pegol. Blue and gray lines indicate those patients who are in favor of damoctocog alfa pegol

and rurioctocog alfa pegol, respectively. $\mathrm{AUC}_{\text {norm }}$, area under the curve normalized for dose per body weight; CL, clearance; $t_{1 / 2}$, half-life

data described earlier using damoctocog alfa and rurioctocog alfa pegol and that the conclusions from this study are robust with respect to the population PK modeling approach pursued.

\section{Discussion}

This is the first randomized head-to-head study conducted to directly compare the PK profiles of two PEGylated EHL rFVIII products, damoctocog alfa pegol and rurioctocog alfa pegol, following a single infusion in patients with severe hemophilia A. Owing to the discrepancy in dosing noted in our study (3\% and $14 \%$ higher than the planned dose of $50 \mathrm{IU} / \mathrm{kg}$ for damoctocog alfa pegol and rurioctocog alfa pegol, respectively), analyses based on actual doses were crucial for a valid,

No adverse events were reported in this study. None of the patients developed antibodies against damoctocog alfa pegol or PEG during the entire study period.

\section{FVIII threshold level}

$1 \%$

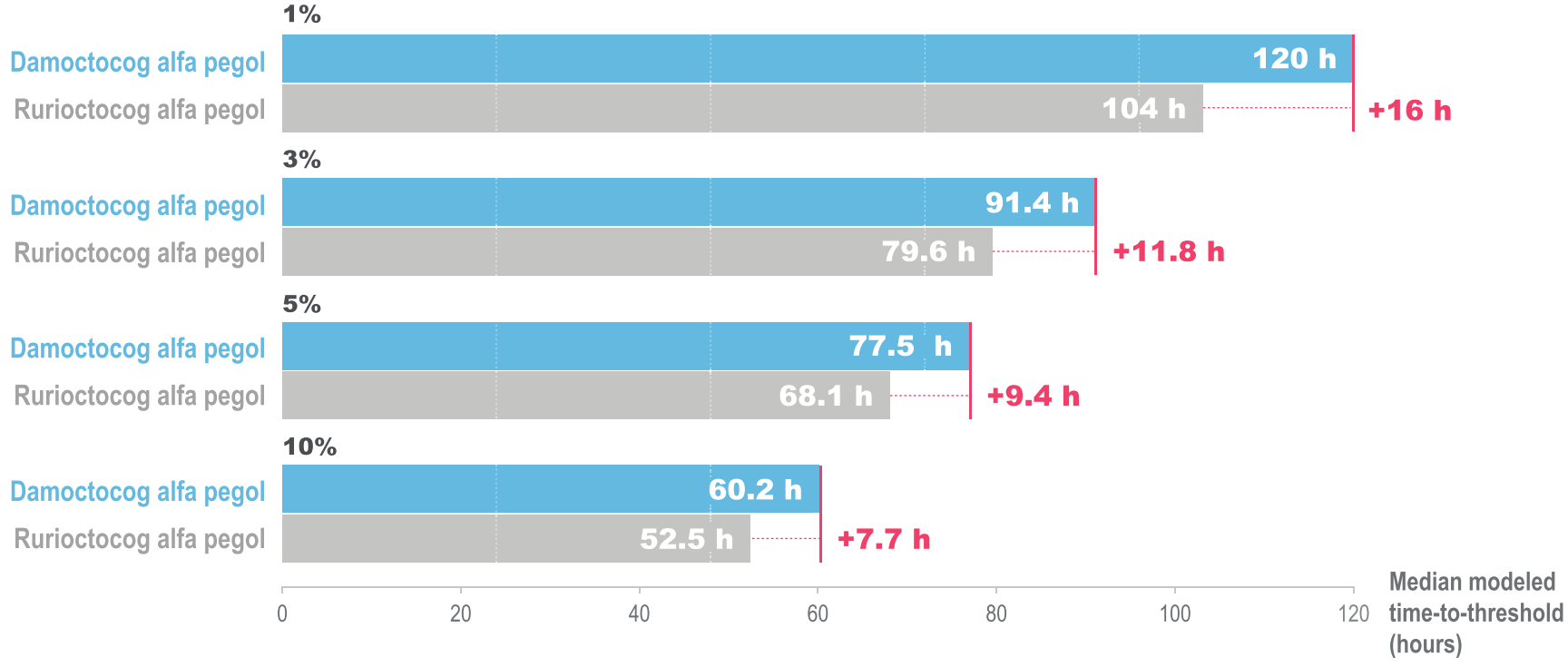

Fig. 3 Median modeled time to FVIII threshold level after a single infusion of $50 \mathrm{IU} / \mathrm{kg}$ damoctocog alfa pegol or rurioctocog alfa pegol. FVIII, factor VIII; h, hours 
Table 3 Parameter estimates of the population PK model

\begin{tabular}{|c|c|c|c|c|}
\hline PK parameter & Value & SE & $\mathrm{CV}$ & $95 \% \mathrm{CI}$ \\
\hline $\mathrm{CL}, \mathrm{dL} / \mathrm{h}$ & 1.63 & 0.159 & 9.75 & $1.32-1.95$ \\
\hline V1, dL & 39.4 & 1.26 & 3.20 & $36.9-41.9$ \\
\hline$Q$ rurioctocog alfa pegol, $\mathrm{dL} / \mathrm{h}$ & 3.00 & 0.726 & 24.2 & $1.58-4.43$ \\
\hline $\mathrm{V} 2$ rurioctocog alfa pegol, $\mathrm{dL}$ & 5.32 & 0.817 & 15.4 & $3.72-6.92$ \\
\hline Relative increase in CL for rurioctocog alfa pegol & 1.21 & 0.0396 & 3.27 & $1.13-1.29$ \\
\hline Interindividual variability in $\mathrm{CL}, \% \mathrm{CV}$ & 41.2 & 0.0615 & 36.3 & $0.0491-0.290$ \\
\hline Interindividual variability in $\mathrm{V} 1, \% \mathrm{CV}$ & 13.0 & 0.00574 & 34.1 & $0.00558-0.0281$ \\
\hline $\begin{array}{l}\text { Interindividual variability in relative increase in CL for rurioctocog alfa pegol } \\
\text { versus damoctocog alfa pegol, \%CV }\end{array}$ & 11.5 & 0.00429 & 32.4 & $0.00484-0.0217$ \\
\hline Interindividual variability for $Q$ rurioctocog alfa pegol, $\% \mathrm{CV}$ & 15.0 (fix) & - & - & - \\
\hline Interindividual variability for $\mathrm{V} 2$ rurioctocog alfa pegol, $\% \mathrm{CV}$ & 15.0 (fix) & - & - & - \\
\hline Residual error (additive) & 0.25 (fix) & - & - & - \\
\hline Residual error (proportional) & 0.117 & 0.00499 & 4.26 & $0.107-0.127$ \\
\hline
\end{tabular}

$C I$, confidence interval; $C L$, clearance; $C V$, coefficient of variation; $L B W$, lean body weight; $P K$, pharmacokinetic; $Q$, intercompartmental clearance; $S E$, standard error; $V$, volume

direct comparison of the PK profiles of damoctocog alfa pegol and rurioctocog alfa pegol. Taking the actual dosing into consideration, damoctocog alfa pegol demonstrated superiority in PK compared with rurioctocog alfa pegol, with a $22 \%$ increase in $\mathrm{AUC}_{\text {norm }}$ compared with rurioctocog alfa pegol, mirrored by a reduction in the clearance of damoctocog alfa pegol (reduced by $18 \%$ ), and a prolonged $t_{1 / 2}$ by $1 \mathrm{~h}(6 \%$ longer) compared with rurioctocog alfa pegol. Importantly, the improvement in PK for damoctocog alfa pegol compared with rurioctocog alfa pegol was observed in most patients in this study.

Improved PK of damoctocog alfa pegol was also observed in a previous head-to-head crossover study, which demonstrated similar levels of improvements in $t_{1 / 2}, \mathrm{CL}$, and AUC for damoctocog alfa pegol compared with $\mathrm{rFVIIIFc}$ following a single infusion in patients with severe hemophilia A [25]. The mean $\mathrm{AUC}_{0-\text { tlast }}$ was $25 \%$ higher for damoctocog alfa pegol, with a $20 \%$ reduction in CL observed for damoctocog alfa pegol compared with rFVIIIFc. Conducted at the same clinical site and using the same one-stage clotting assay as the current study, similar PK values for damoctocog alfa pegol were also observed with, for example, a $t_{1 / 2}$ for damoctocog alfa pegol of $16.3 \mathrm{~h}$ compared with that reported in the current study $(17.0 \mathrm{~h})$. Results from Shah et al. and the current study are indicative of the consistency in improvement of PK parameters for damoctocog alfa pegol, compared with the respective comparator EHL FVIII products rFVIIIFc and rurioctocog alfa pegol. The improvements observed for AUC and CL, translating to prolonged time-to-threshold, also suggest that these PK parameters may be more valuable to consider than the relatively small, albeit significant, differences in half-life reported, when exploring differences in the PK profiles of EHL rFVIII products.
As patients present with varied clinical phenotypes, tailored patient therapy should be guided by careful consideration of the overall improved PK profile of an EHL rFVIII product $[24,30]$. Besides half-life, PK parameters such as AUC, CL, and time above FVIII threshold level are important contributors to protection from bleeds and, thus, are of clinical significance [31]. In patients with severe hemophilia A, time spent with very low FVIII levels potentially carries the risk of spontaneous joint bleeds [3]. Additionally, duration of treatment effect has direct implications in dosing frequency and related prophylaxis adherence barriers [32]. In this regard, in the current study, the median time to reach $1 \mathrm{IU} / \mathrm{dL}$ was $16 \mathrm{~h}$ longer for damoctocog alfa pegol compared with rurioctocog alfa pegol. Taken together, the superior PK profile observed for damoctocog alfa pegol, compared with rurioctocog alfa pegol, in the current study has potential clinical significance in relation to protection from bleeds in patients with hemophilia A. These data suggest patients may benefit from the higher FVIII activity levels and longer time to reach thresholds achieved with damoctocog alfa pegol compared with rurioctocog alfa pegol, using similar doses and dose intervals. Thus, bleed protection can potentially be improved at the same dosing intervals, or existing protection level can be maintained from less frequent infusions with damoctocog alfa pegol.

Properties of the conjugated PEG are known to be driving factors that influence the PK properties of PEGylated proteins [33]. Both the size of the PEG molecule and the type of conjugation are thought to play a role, and it is hypothesized that the differences in these features employed in damoctocog alfa pegol and rurioctocog alfa pegol could contribute to the superior PK profile of damoctocog alfa pegol observed in the current study. Animal models have demonstrated that terminal 
$t_{1 / 2}$ is affected by PEG size. In hemophilia A mouse and rabbit models, increased conjugated PEG size was associated with an increased terminal $t_{1 / 2}: 9.8 \mathrm{~h}$ and up to $13.6 \mathrm{~h}$ with $30 \mathrm{kDa}$ and $60 \mathrm{kDa}$ PEGylated FVIII, respectively [11]. PEGylation in damoctocog alfa pegol is achieved by a targeted approach of site-specific conjugation at a single site in the BDD rFVIII molecule $[11,34]$. Site-specific PEGylation may help to avoid loss of activity or alter protein dynamics due to steric hindrance from extensive PEGylation. The choice of PEGylation site has been shown to influence the activity and molecular dynamics of the conjugated product in other studies $[35,36]$. The PEGylation process utilized in rurioctocog alfa pegol, on the other hand, targets the B-domain and thus can introduce PEGylation nonuniformly across the whole length of the B-domain in rFVIII protein [12], including potential unfavorable sites that may affect the molecular dynamics of rurioctocog alfa.

One limitation of the current study was that it was only conducted in adults aged 18-65 years. However, no major differences were observed in the PK profiles between adults and adolescents for damoctocog alfa pegol and rurioctocog alfa pegol [21,37]. Based on the observation by Shah et al., the improvement in PK observed in this comparison of damoctocog alfa pegol versus rurioctocog alfa pegol should also occur in adolescents [21]. The small size of the patient cohort and the single-center study design could be another potential limitation of this study. However, single-center crossover studies are appropriate for meeting the objectives of this study. A major strength of this study was its crossover design, used to overcome issues around interpatient variability in PK. Intertrial comparison of PK can be inaccurate and inconclusive due to confounding factors such as interpatient variability. Contributing factors for PK variability include interpatient heterogeneity in variables such as von Willebrand factor, age, body size, and blood group [28, 38]. Due to these interpatient variations, a parallel study design or attempts on intertrial comparisons based on previously published data for damoctocog alfa pegol and rurioctocog alfa pegol could result in inaccurate assessment of comparison [22]. The crossover design used in this study thus eliminates the pitfalls associated with confounding factors, as the PK parameters were evaluated using the same assay in the same patient population $[22,23]$. Additionally, similarities in the damoctocog alfa pegol PK profile between the current study and the previous head-to-head study by Shah et al. indicate consistency in the modeling process [25]. Another strength of this study was that the data were analyzed using different analysis approaches, including noncompartmental and compartmental methods (popPK). Irrespective of the approach utilized, the findings were the same in that significant differences in PK were detected between damoctocog alfa pegol and rurioctocog alfa pegol, in favor of damoctocog alfa pegol.
In conclusion, damoctocog alfa pegol had a superior PK profile compared with rurioctocog alfa pegol, including a higher $\mathrm{AUC}_{\mathrm{norm}}$ (based on potency-adjusted analyses), extended half-life, and a longer median time to reach $1 \% \mathrm{IU} /$ dL FVIII (based on popPK modeling) following a single infusion in patients with severe hemophilia A.

Acknowledgments The authors thank the following personnel from Bayer: Jean Allen, Joseph Pagnotta, Gani Ivanov, and Hristo Hristov (study management); David Banczyk and Eva Busemann (bioanalytics); Christoph Luhn (PK evaluation); and Katrin Walkamp and Nina Besche (statistics). The authors also thank Peter Vis from LAP\&P Consultants BV (Leiden, the Netherlands) for assistance with the popPK analysis.

Authors' contributions Alexander Solms provided substantial contribution to the study design, implementation, and data analysis. Anita Shah also provided substantial contribution to the data analysis. Erik Berntorp, Andreas Tiede, Alfonso Iorio, Camila Linardi, and Maria Elisa Mancuso provided considerable input into the data interpretation. Camila Linardi also participated in the study conduct and contributed to protocol and study report preparations. Maurice Ahsman supported the popPK and modeling simulation. Toshko Lissitchkov and his colleague Tihomir Zhivkov were the lead and subinvestigators, respectively. All authors provided input into drafting and revision of this manuscript, and all agree to be accountable for all aspects of the work in ensuring that questions related to the accuracy or integrity of any part of the work are appropriately investigated and resolved.

Funding This study was funded by Bayer AG, Leverkusen, Germany. Medical writing assistance was provided by Sreerekha S. Pillai, $\mathrm{PhD}$, of Darwin Healthcare Communications, fully funded by Bayer.

\section{Compliance with ethical standards}

Conflict of interest Alexander Solms is a Bayer employee and shareholder; Anita Shah is a Bayer employee and shareholder. Erik Berntorp has received grants/research support from Bayer, CSL Behring, Shire, and Sobi/Bioverativ; honoraria/consultation fee from Bayer, Octapharma, and Shire/Takeda; and speaker fees from Bayer. Andreas Tiede has received grants/research support from Biotest, Novo Nordisk, Octapharma, Roche, and Takeda and honoraria/consultation fee from Bayer, Biotest, Chugai, CSL Behring, Novo Nordisk, Octapharma, Roche, and Takeda. Alfonso Iorio has received grants/research support through from Bayer, CSL Behring, Grifols, Novo Nordisk, Octapharma, Pfizer, Roche, and Shire; Camila Linardi is a Bayer employee; Maurice Ahsman is a consultant for Bayer; Maria Elisa Mancuso has received honoraria/consultation fee from Bayer, Bioverativ, Catalyst, CSL Behring, Grifols, Kedrion, Novo Nordisk, Octapharma, Pfizer, Roche, Shire, and Sobi and received speaker fees from Bayer, Biotest, CSL Behring, Grifols, Kedrion, Novo Nordisk, Octapharma, Pfizer, Roche, Shire, and Sobi; Tihomir Zhivkov has received grants/research support from Bayer and is a subinvestigator of clinical trials for Apellis, Bayer, Catalyst, Octapharma, and Sanofi. Toshko Lissitchkov has received honoraria/consultation fee for advisory boards from Bayer, Roche, and Sobi; received speaker fees from Bayer, Novo Nordisk, Roche, and Sobi; and is a principal investigator of clinical trials sponsored by Bayer, CSL Behring, Biotest, Novo Nordisk, Octapharma, Pfizer, Rigel, SanofiAlnylam, and Sanofi-Bioverativ.

Ethical approval The study was approved by the institutional review board at the single site and was carried out in compliance with the 
protocol, the principles of the 1975 Declaration of Helsinki and its later amendments, and good clinical practice guidelines.

Consent to participate All patients gave written informed consent before initiation of any study-related procedures.

\section{Consent for publication Not applicable}

Code availability Not applicable

Open Access This article is licensed under a Creative Commons Attribution 4.0 International License, which permits use, sharing, adaptation, distribution and reproduction in any medium or format, as long as you give appropriate credit to the original author(s) and the source, provide a link to the Creative Commons licence, and indicate if changes were made. The images or other third party material in this article are included in the article's Creative Commons licence, unless indicated otherwise in a credit line to the material. If material is not included in the article's Creative Commons licence and your intended use is not permitted by statutory regulation or exceeds the permitted use, you will need to obtain permission directly from the copyright holder. To view a copy of this licence, visit http://creativecommons.org/licenses/by/4.0/.

\section{References}

1. Srivastava A, Brewer AK, Mauser-Bunschoten EP, Key NS, Kitchen S, Llinas A, Ludlam CA, Mahlangu JN, Mulder K, Poon MC, Street A, Hemophilia TGWGoBoTWFO (2013) Guidelines for the management of hemophilia. Haemophilia 19(1):e1-47. https://doi.org/10.1111/j.1365-2516.2012.02909.x

2. Iorio A, Marchesini E, Marcucci M, Stobart K, Chan AKC (2011) Clotting factor concentrates given to prevent bleeding and bleeding-related complications in people with hemophilia A or B. Cochrane Database Syst Rev 9. https://doi.org/10.1002/14651858. CD003429.pub4

3. Collins PW, Bjorkman S, Fischer K, Blanchette V, Oh M, Schroth P, Fritsch S, Casey K, Spotts G, Ewenstein BM (2010) Factor VIII requirement to maintain a target plasma level in the prophylactic treatment of severe hemophilia A: influences of variance in pharmacokinetics and treatment regimens. J Thromb Haemost 8(2): 269-275. https://doi.org/10.1111/j.1538-7836.2009.03703.x

4. Khair K, Lawrence K, Butler R, Butler R, O'Shea E, Christie BA (2008) Assessment of treatment practice patterns for severe hemophilia A: a global nurse perspective. 1421-9662 (Electronic))

5. Geraghty S, Dunkley T, Harrington C, Lindvall K, Maahs J, Sek J (2006) Practice patterns in haemophilia A therapy - global progress towards optimal care. Haemophilia 12(1):75-81. https://doi.org/10. 1111/j.1365-2516.2006.01189.x

6. Manco-Johnson MJ, Abshire TC, Shapiro AD, Riske B, Hacker MR, Kilcoyne R, Ingram JD, Manco-Johnson ML, Funk S, Jacobson L, Valentino LA, Hoots WK, Buchanan GR, DiMichele D, Recht M, Brown D, Leissinger C, Bleak S, Cohen A, Mathew P, Matsunaga A, Medeiros D, Nugent D, Thomas GA, Thompson AA, McRedmond K, Soucie JM, Austin H, Evatt BL (2007) Prophylaxis versus episodic treatment to prevent joint disease in boys with severe hemophilia. N Engl J Med 357(6):9

7. Oldenburg J (2015) Optimal treatment strategies for hemophilia: achievements and limitations of current prophylactic regimens. Blood 125(13):2038-2044. https://doi.org/10.1182/blood-201501-528414
8. Graf L (2018) Extended half-life factor VIII and factor IX preparations. Transfus Med Hemother 45(2):86-91. https://doi.org/10. $1159 / 000488060$

9. Mahdi AJ, Obaji SG, Collins PW (2015) Role of enhanced half-life factor VIII and IX in the treatment of haemophilia. Br J Haematol 169(6):768-776. https://doi.org/10.1111/bjh.13360

10. Pipe SW (2016) New therapies for hemophilia. Hematology Am Soc Hematol Educ Program 2016(1):650-656. https://doi.org/10. 1182/asheducation-2016.1.650

11. Mei B, Pan C, Jiang H, Tjandra H, Strauss J, Chen Y, Liu T, Zhang X, Severs J, Newgren J, Chen J, Gu JM, Subramanyam B, Fournel MA, Pierce GF, Murphy JE (2010) Rational design of a fully active, long-acting PEGylated factor VIII for hemophilia A treatment. Blood 116(2):270-279. https://doi.org/10.1182/blood-2009-11254755

12. Turecek PL, Bossard MJ, Graninger M, Gritsch H, Höllriegl W, Kaliwoda M, Matthiessen P, Mitterer A, Muchitsch EM, Purtscher M, Rottensteiner H, Schiviz A, Schrenk G, Siekmann J, Varadi K, Riley T, Ehrlich HJ, Schwarz HP, Scheiflinger F (2012) BAX 855, a PEGylated rFVIII product with prolonged half-life. Development, functional and structural characterisation. Hamostaseologie 32(Suppl 1):S29-S38

13. Valentino LA, Cong L, Enockson C, Song X, Scheiflinger F, Muchitsch EM, Turecek PL, Hakobyan N (2015) The biological efficacy profile of BAX 855, a PEGylated recombinant factor VIII molecule. Haemophilia 21(1):58-63. https://doi.org/10.1111/hae. 12532

14. Lalezari S, Reding MT, Pabinger I, Holme PA, Negrier C, Chalasani P, Shin HJ, Wang M, Tseneklidou-Stoeter D, Maas Enriquez M (2019) BAY 94-9027 prophylaxis is efficacious and well tolerated for up to $>5$ years with extended dosing intervals: PROTECT VIII extension interim results. Haemophilia. https://doi. org/10.1111/hae.13853

15. Santagostino E, Lalezari S, Reding MT, Ducore J, Ng HJ, Poulsen LH, Michaels LA, Linardi CCG (2019) Safety and efficacy of BAY 94-9027, an extended-half-life factor VIII, during surgery in patients with severe hemophilia A: results of the PROTECT VIII clinical trial. Thromb Res 183:13-19. https://doi.org/10.1016/j. thromres.2019.08.023

16. Brand B, Gruppo R, Wynn TT, Griskevicius L, Lopez Fernandez MF, Chapman M, Dvorak T, Pavlova BG, Abbuehl BE (2016) Efficacy and safety of pegylated full-length recombinant factor VIII with extended half-life for perioperative haemostasis in haemophilia A patients. Haemophilia 22(4):e251-e258. https:// doi.org/10.1111/hae.12963

17. Konkle BA, Stasyshyn O, Chowdary P, Bevan DH, Mant T, Shima M, Engl W, Dyck-Jones J, Fuerlinger M, Patrone L, Ewenstein B, Abbuehl B (2015) Pegylated, full-length, recombinant factor VIII for prophylactic and on-demand treatment of severe hemophilia A. Blood 126(9):1078-1085. https://doi.org/10.1182/blood-2015-03630897

18. U.S. Food and Drug Administration (2018) Summary basis for regulatory action https://www.fda.gov/media/116286/download. Accessed 28 April 2020

19. Baxalta US Inc (2018) Adynovate - prescribing information. Bannockburn, IL, USA. https://www.shirecontent.com/PI/PDFs/ ADYNOVATE_USA_ENG.pdf. Accessed 10 April 2020

20. Coyle TE, Reding MT, Lin JC, Michaels LA, Shah A, Powell J (2014) Phase I study of BAY 94-9027, a PEGylated B-domaindeleted recombinant factor VIII with an extended half-life, in subjects with hemophilia A. J Thromb Haemost 12(4):488-496

21. Shah A, Coyle T, Lalezari S, Fischer K, Kohlstaedde B, Delesen H, Radke S, Michaels LA (2018) BAY 94-9027, a PEGylated recombinant factor VIII, exhibits a prolonged half-life and higher area under the curve in patients with severe haemophilia A: 
comprehensive pharmacokinetic assessment from clinical studies. Haemophilia 24(5):733-740. https://doi.org/10.1111/hae.13561

22. Song F, Loke YK, Walsh T, Glenny A-M, Eastwood AJ, Altman DG (2009) Methodological problems in the use of indirect comparisons for evaluating healthcare interventions: survey of published systematic reviews. BMJ 338:b1147. https://doi.org/10.1136/bmj. b1147

23. Evans SR (2010) Clinical trial structures. J Exp Stroke Transl Med 3(1):8-18. https://doi.org/10.6030/1939-067x-3.1.8

24. Iorio A, Edginton AN, Blanchette V, Blatny J, Boban A, Cnossen M, Collins P, Croteau SE, Fischer K, Hart DP, Ito S, Korth-Bradley J, Lethagen S, Lillicrap D, Makris M, Mathôt R, Morfini M, Neufeld EJ, Spears J (2018) Performing and interpreting individual pharmacokinetic profiles in patients with hemophilia A or B: rationale and general considerations. Res Pract Thromb Haemost 2(3): 535-548. https://doi.org/10.1002/rth2.12106

25. Shah A, Solms A, Wiegmann S, Ahsman M, Berntorp E, Tiede A, Iorio A, Mancuso ME, Zhivkov T, Lissitchkov T (2019) Direct comparison of two extended-half-life recombinant FVIII products: a randomized, crossover pharmacokinetic study in patients with severe hemophilia A. Ann Hematol 98(9):2035-2044. https://doi. org/10.1007/s00277-019-03747-2

26. Carcao MD, Chelle P, Clarke E, Kim L, Tiseo L, Morfini M, Hossain T, Rand ML, Brown C, Edginton AN, Lillicrap D, Iorio A, Blanchette VS (2019) Comparative pharmacokinetics of two extended half-life FVIII concentrates (Eloctate and Adynovate) in adolescents with hemophilia $\mathrm{A}$ : is there a difference? J Thromb Haemost 17(7):1085-1096. https://doi.org/10.1111/jth.14469

27. Garmann D, McLeay S, Shah A, Vis P, Maas Enriquez M, Ploeger BA (2017) Population pharmacokinetic characterization of BAY 81-8973, a full-length recombinant factor VIII: lessons learned importance of including samples with factor VIII levels below the quantitation limit. Haemophilia 23(4):528-537. https://doi.org/10. 1111/hae.13192

28. Solms A, Iorio A, Ahsman MJ, Vis P, Shah A, Berntorp E, Garmann D (2020) Favorable pharmacokinetic characteristics of extended-half-life recombinant factor VIII BAY 94-9027 enable robust individual profiling using a population pharmacokinetic approach. Clin Pharmacokinet 59(5):605-616. https://doi.org/10. 1007/s40262-019-00832-7

29. Chelle P, Yeung CHT, Croteau SE, Lissick J, Balasa V, Ashburner C, Park YS, Bonanad S, Megías-Vericat JE, Nagao A, Wynn T, Corrales-Medina F, Tran H, Sharathkumar A, Chitlur M, Sarmiento S, Edginton A, Iorio AA-O (2020) Development and validation of a population-pharmacokinetic model for rurioctacog alfa pegol (Adynovate $(\circledR)$ ): a report on behalf of the WAPPS-Hemo Investigators Ad Hoc Subgroup. (1179-1926 (Electronic))

30. Iorio A, Blanchette V, Blatny J, Collins P, Fischer K, Neufeld E (2017) Estimating and interpreting the pharmacokinetic profiles of individual patients with hemophilia $\mathrm{A}$ or B using a population pharmacokinetic approach: communication from the SSC of the ISTH. J Thromb Haemost 15(12):2461-2465. https://doi.org/10.1111/jth. 13867

31. Morfini M, Gherardini S (2018) Pharmacokinetic-based prediction of real-life dosing of extended half-life clotting factor concentrates on hemophilia. Ther Adv Hematol 9(6):149-162. https://doi.org/ $10.1177 / 2040620718774258$

32. Thornburg CD, Duncan NA (2017) Treatment adherence in hemophilia. Patient Prefer Adherence 11:1677-1686. https://doi.org/10. 2147/PPA.S139851

33. Baumann A (2020) PEGylated biologics in haemophilia treatment: current understanding of their long-term safety. Haemophilia 26(1): e11-e13. https://doi.org/10.1111/hae.13875

34. Ivens IA, Baumann A, McDonald TA, Humphries TJ, Michaels LA, Mathew P (2013) PEGylated therapeutic proteins for haemophilia treatment: a review for haemophilia caregivers. Haemophilia 19(1):11-20. https://doi.org/10.1111/j.1365-2516. 2012.02931.x

35. $\mathrm{Mu} \mathrm{Q}, \mathrm{Hu} \mathrm{T}, \mathrm{Yu} \mathrm{J}$ (2013) Molecular insight into the steric shielding effect of PEG on the conjugated staphylokinase: biochemical characterization and molecular dynamics simulation. PLoS One 8(7): e68559-e68559. https://doi.org/10.1371/journal.pone.0068559

36. Kaur N, Sinha PK, Sahni G (2019) Site-specific PEGylation of micro-plasmin for improved thrombolytic therapy through engineering enhanced resistance against serpin mediated inhibition. PLoS One 14(5):e0217234. https://doi.org/10.1371/journal.pone. 0217234

37. Baxalta US Inc (2015) Adynovate-prescribing information

38. Lalezari S, Martinowitz U, Windyga J, Enriquez MM, Delesen H, Schwartz L, Scharrer I (2014) Correlation between endogenous VWF:Ag and PK parameters and bleeding frequency in severe haemophilia A subjects during three-times-weekly prophylaxis with rFVIII-FS. Haemophilia 20(1):e15-e22. https://doi.org/10. 1111/hae.12294

Publisher's note Springer Nature remains neutral with regard to jurisdictional claims in published maps and institutional affiliations. 